\title{
Epilepsia de Ausência na Infância e seu Impacto na Aprendizagem
}

\author{
Childhood Absence Epilepsy and Its Impact on Learning
}

\section{Roberta de Figueiredo Gomes', Angela Maria de Freitas', Adriana Machado Vasques", Adriana Gutterres Pereira', Eloisa Elena Silveira Ferreira $^{5}$, Mirna WettersPortuguez ${ }^{6}$}

\section{RESUMO}

Introduçáo. A epilepsia de ausência na infância é um distúrbio neurológico que causa importantes dificuldades biopsicossociais e um atraso no desenvolvimento da aprendizagem. Objetivo. Descrever a importância do diagnóstico precoce e verificar fatores que impactam na aprendizagem em crianças com epilepsia de ausência. Método. Revisão de literatura pertinente acerca da epilepsia de ausência na infância, seu impacto na aprendizagem e respectivas repercussóes. Conclusáo. Ainda que a epilepsia de ausência possa ser uma condição comum encontrada em crianças, geralmente o seu diagnóstico não costuma ser fácil, o que pode levar a dificuldades no desempenho da aprendizagem. Diante disso, torna-se fundamental que os pais e profissionais envolvidos com a criança tenham pleno conhecimento sobre sua condição, sobre seus aspectos biopsicossociais e que estejam atentos aos sinais de alarme da epilepsia de ausência.

Unitermos. Epilepsia de Ausência na Infância, Aprendizagem, Educação.

Citaçáo. Gomes RF, Freitas AM, Vasques AM, Pereira AG, Ferreira EES, WettersPortuguez M. Epilepsia de Ausência na Infância e seu Impacto na Aprendizagem.

\begin{abstract}
Introduction. The childhood absence epilepsy is a neurological disorder that causes a significant biopsychosocial difficulties and a delay in development of learning. Objective. To describe the importance of early diagnosis and identify factors that impact on learning in children with absence epilepsy. Method. Review of literature about childhood absence epilepsy, its impact and effects on learning. Conclusions. Although absence epilepsy can be a common condition found in children, usually the diagnosis is not easy, which can lead to difficulties in learning performance. Therefore, it is essential that parents and professionals involved with the child should be fully aware of its condition on biopsychosocial aspects and to be alert to warning signs of absence epilepsy.
\end{abstract}

Keywords. Absence Epilepsy, Learning, Education.

Citation. Gomes RF, Freitas AM, Vasques AM, Pereira AG, Ferreira EES, WettersPortuguez M. Childhood Absence Epilepsy and Its Impact on Learning.
Trabalho realizado na Pontifícia Universidade Católica do Rio Grande do Sul - PUCRS, Porto Alegre-RS, Brasil.

1.Neuropsicóloga, Mestre e Doutoranda em Medicina e Ciências da SaúdeNeurociências pela Pontifícia Universidade Católica do Rio Grande do SulPUCRS, Porto Alegre-RS, Brasil.

2.Psicóloga, Mestre e Doutoranda em Medicina e Ciências da Saúde- Neurociências pela Pontifícia Universidade Católica do Rio Grande do Sul- PUCRS, Porto Alegre-RS, Brasil.

3.Psicóloga, Mestre e Doutoranda em Geriatria e Gerontologia pela Pontifícia Universidade Católica do Rio Grande do Sul-PUCRS, Porto Alegre-RS, Brasil. 4.Psicóloga, Mestre e Doutora em Medicina e Ciências da Saúde - Neurociências pela Pontifícia Universidade Católica do Rio Grande do Sul-PUCRS, Porto Alegre-RS, Brasil.

5.Terapeuta Ocupacional, Mestre e Doutoraem Medicina e Ciências da SaúdeNeurociências pela Pontifícia Universidade Católica do Rio Grande do SulPUCRS, Porto Alegre-RS, Brasil.

6.Neuropsicóloga, Doutora em Neurociências pela Universidade Federal de Sáo Paulo- UNIFESP, professora adjunta da Pontifícia Universidade Católica do Rio Grande do Sul-PUCRS, Porto Alegre-RS, Brasil.
Endereço para correspondência: Mirna WettersPortuguez Avenida Ipiranga, 6681 CEP 90619-900, Porto Alegre-RS, Brasil. E-mail:mirna@pucrs.br 


\section{INTRODUÇÃO}

A denominação epilepsia provém da palavra grega epilambaneima qual significa surpresa. É considerada uma doença crônica, um sinal ou sintoma de um distúrbio neurológico que geralmente se manifesta sob a forma de crises convulsivas recorrentes com grau variável de intensidade e duração ${ }^{1}$.

As crises convulsivas resultam de uma descarga excessiva de neurônios em determinada área cerebral que repercutem diretamente na cognição, linguagem e escolarização².

Estima-se que existam em torno de 60 milhóes de pessoas com epilepsia no mundo, enquanto no Brasil, a estimativa é que a epilepsia acometa $1-2 \%$ da população ${ }^{3}$. Sua incidência varia conforme a idade, porém crianças são mais suscetíveis devido a sensibilidade do cérebro ${ }^{4}$.

Com estes índices de prevalência associados a grupos vulneráveis, impacto socioeconômico e uma maior morbimortalidade, a epilepsia tem sido considerada um problema de saúde pública.

Dentre as epilepsias mais comuns na infância, está a epilepsia de ausência ${ }^{5,6}$. Este tipo de epilepsia merece atenção especial devido à infância ser um período especialmente importante para se reconhecer sinais de alarme como problemas cognitivos e emocionais que podem estar ligados à doença, permitindo intervenção precoce e redução do dano biopsicossocial associado às doenças crônicas em geral e da epilepsia em particular. Geralmente este tipo de epilepsia tem início entre os quatro e sete anos de idade e, com o ingresso na escola, começam a serem detectados os primeiros problemas de aprendizagem. O aparecimento das crises antes dos três anos é raro ${ }^{7}$.

A ausência de literatura que aborde o contexto escolar da criança portadora de epilepsia no tocante às suas necessidades, a escassez de trabalhos voltados para essa perspectiva, assim como a falta de informaçóes para o manejo e assistência adequada levam ao atraso no diagnóstico e tratamento da epilepsia, contribuindo para maiores danos e dificuldades cognitivas da criança com EAI.

Devido à alta incidência de epilepsia na idade escolar, é fundamental atentar-se para o atendimento das crianças em suas necessidades básicas de natureza física, emocional e social. Deste modo, o objetivo central do artigo é descrever a importância do diagnóstico precoce e verificar fatores que impactam na aprendizagem em crianças com epilepsia de ausência.

\section{MÉTODO}

Este é um artigo de revisão de literatura realizado através de pesquisas de artigos e jornais indexados no Medline, Scielo e liga Brasileira de Epilepsia. Nesta pesquisa de artigos os descritores utilizados foram epilepsia de ausência na infância, aprendizagem e desenvolvimento cognitivo. Os estudos analisados foram os que ocorreram do ano de 2006 a 2012, tanto na América Latina, como América do Norte e Europa. Os critérios de inclusão dos textos selecionados referem-se à abordagem direcionada para a questão da EAI e os efeitos diretos e indiretos na aprendizagem de crianças de 6 a 12 anos. Foram excluídos todos os artigos que apresentavam a abordagem EAI que não apresentavam abordagem com educação e aprendizagem. Foram acessados aproximadamente 70 artigos em um período de 2 meses, sendo utilizados 27.

\section{Epilepsia de Ausência na Infância}

A epilepsia de ausência da infância (EAI) é uma epilepsia generalizada idiopática ${ }^{8}$, responsável por $10 \mathrm{a}$ $12 \%$ das epilepsias em crianças com idades inferiores a 16 anos de idade. Caracteriza-se por uma breve perda de consciência que tem duração de 3 a 10 segundos, podendo ter uma ocorrência de até 200 crises diárias ${ }^{9,10}$.

Considera-se essa forma de epilepsia com bom prognóstico em longo prazo. No entanto, uma parte das crianças (44\%) não apresentará remissão completa das crises, evoluindo para outra forma de epilepsia generalizada (mioclônica juvenil). Sendo assim, faz-se necessário o acompanhamento desses pacientes por um longo tempo ${ }^{11}$.

Sendo a EAI a forma de epilepsia mais comum entre crianças, muitos estudos estão procurando analisar até que medida há prejuízos na aprendizagem e até que ponto esta dificuldade pode ser atribuída às descargas interictaisepileptiformes ${ }^{12}$.

Estudos demonstram que muitos pacientes apresentam desenvolvimento neuropsicomotor normal ${ }^{7}$. Porém, existem evidências de que pode haver comprometimento das funçóes cognitivas e do comportamento, assim como o aparecimento de transtornos psiquiátricos ${ }^{13}$.

Como a etiologia é multifatorial, a aprendizagem 
acaba sofrendo influência do tipo de epilepsia, da sua causa, da frequência com que acontecem as crises, se há história prévia de estado de mal epiléptico e do papel das descargas epileptiformesinterictais. Estas descargas parecem estar diretamente ligadas ao comprometimento cognitivo e comportamental ${ }^{12}$.

De acordo com Pardoe et al. ${ }^{14}$, existe uma redução do volume da substância cinzenta subcortical e no tálamo e um aumento da substância branca no lobo frontal e lobo temporal direito em adolescentes e jovens adultos com epilepsia de ausência. Estes achados neuroanatômicos são cruciais para complementar os estudos em pacientes que apresentam dificuldades de aprendizagem.

\section{Impacto na Aprendizagem}

A aprendizagem refere-se a mudança comportamental viabilizada pela plasticidade dos processos neurais cognitivos e as alteraçóes neste processo levam à dificuldades de aprendizagem que acarretam prejuízos consideráveis, tais como distúrbios motores, psicomotores, na atenção, memorização, desinteresse e problemas de comportamento $^{15}$.

Dentre os processos básicos das funçóes cognitivas, a atenção é responsável pela conexão entre a percepção e o processamento de informaçấo. Ela também influencia e modula outras funçóes cognitivas, como as funçóes executivas $^{16,17}$.

Existe uma forte evidência de maior prevalência de Transtorno de Déficit de Atenção e/ou Hiperatividade (TDAH) e de problemas de atenção em crianças com epilepsia. A possível explicação é que o desenvolvimento cognitivo, incluindo atenção, é complexo e está atingido pela interação de distintas variáveis, como a atividade convulsiva, a medicação, lugar e extensão da disfunção cerebral subjacente, idade das primeiras crises, fatores ambientais, emocionais e genética ${ }^{18}$.

Estudos têm associado a EAI à déficits de atenção e funções executivas. No estudo de D'Agati et al. (2012), ao comparar crianças de 8 a 15 anos com epilepsia de ausência com controles saudáveis, foram encontradas diferenças significativas no tempo total de planejamento de tarefas, fonológica e fluência verbal e atenção sustentada e dividida ${ }^{19}$.

Atualmente, existem muitos indícios de que a epilepsia na infância compromete o desenvolvimento cognitivo e, em uma pequena população dessas crianças, há uma deterioração cognitiva global ${ }^{20,21}$. As descargas epileptiformes parecem estar associadas com distúrbios neuropsicológicos como déficits cognitivos e problemas comportamentais, mesmo que nâo haja evidência de manifestação clínica.

Relatórios preliminares não controlados e três estudos randomizados controlados de tratamento antiepiléptico de descargas interictais sugerem que suprimir as descargas pode melhorar significativamente a função psicossocial. Entretanto, mais estudos devem ser realizados para haver confirmação desta hipótese.

Conforme descrito na literatura, as dificuldades escolares geralmente relacionam-se ao próprio distúrbio e inclui idade de início, frequência, grau, controle das crises, etiologia e tipo de medicação utilizada. Um estudo que abrangeu uma investigação em escolas da Grécia mostrou que $100 \%$ das sessenta e duas crianças que participaram da amostra faltaram às aulas, com uma média de três faltas em um período de seis meses. Ainda, $53,2 \%$ não realizavam as atividades esportivas pela razão de os pais apresentarem excessiva preocupação com a possibilidade de ocorrência de crises na escola ${ }^{22,23}$. Em algumas crianças, as dificuldades de atenção parecem ser o primeiro indício de que existe um déficit cognitivo causado pelas convulsóes ${ }^{24}$. Além destes fatores, podem estar envolvidos aspectos psicossociais como baixa expectativa dos pais e professores quanto ao desempenho da criança, a possibilidade de ser rejeitada pelos colegas e professores, baixa autoestima, funcionamento familiar e redes de apoio social dos pais.

Outro estudo brasileiro com cinquenta crianças cujo objetivo foi avaliar o impacto das crises epilépticas no período escolar mostrou que $80 \%$ delas já tinham faltado pelo menos um dia na escola por causa das crises. Os pais (46\%), afirmaram que se o filho tivesse uma crise, imeditamente iria deixar a escola e $60 \%$ das famílias permitiam que seus filhos não fossem a escola mesmo sem a ocorrência de crises no dia ${ }^{25}$.

Pode-se concluir, através destes estudos, que o grau de conhecimento sobre a epilepsia influencia as atitudes referidas aos pacientes portadores dessa patologia. Os aspectos biopsicossociais parecem ser mais impactantes nas 
dificuldades de aprendizagem, isto é, os problemas cognitivos associados à EAI podem ser mais caracterizados por problemas intrínsecos a condição do que ao distúrbio propriamente dito.

Juntamente com os aspectos biopsicossociais, fator que influencia o desenvolvimento da aprendizagem é o tipo de epilepsia. Um estudo demonstrou que pacientes com crises generalizadas apresentaram escore de inteligência mais baixo do que pacientes com crises focais ${ }^{26}$. Outro fator que apresenta influência é o tratamento medicamentoso. Crianças tratadas com alguns antiepilépticos podem apresentar efeitos colaterais, mas muitas vezes estes são transitórios e não requerem a interrupção do tratamento. A associação entre as dificuldades de aprendizagem e medicação devem ser cuidadosamente analisados, pois dependem de diferentes perfis metabólicos, interações com outras medicaçóes e dos efeitos adversos cognitivos que uma porcentagem de pacientes apresenta ${ }^{27}$.

Dessa forma, o reconhecimento de sinais que possam indicar alguma anormalidade, assim como a divulgação pelos canais competentes (associaçóes médicas ou educacionais) que revelem informaçóes mais consistentes acerca da EAI faz-se necessário, tanto para que os pais, como os professores possam servir de agentes identificadores do problema, permitindo o encaminhamento aos profissionais habilitados a realizar avaliação e diagnóstico. Caplan et al. ${ }^{28}$ e Jones et al. ${ }^{29}$ ressaltam a importância de identificação e tratamento da EAI precocemente, pois com o passar do tempo este distúrbio começa a apresentar um amplo espectro de comorbidades, e a existência de déficit de atenção e das funçôes executivas geram dificuldades que vão desde as atividades de vida diária, até declínios na aprendizagem.

Como se pode observar, muitos fatores são responsáveis pela relação entre epilepsia e baixo desempenho cognitivo em EAI, e quanto mais cedo existir intervenção, menor probabilidade de acontecerem déficits funcionais cumulativos, evitando em longo prazo o baixo rendimento escolar e laboral em adultos com este tipo de epilepsia.

\section{CONSIDERAÇÕES FINAIS}

Como a EAI trata-se de uma condição comum, mas não bem diagnosticada e tratada, pode apresentar repercussóes biopsicossociais para as crianças, interferindo no desenvolvimento infantil e piora na qualidade de vida, já que a criança pode apresentar dificuldades de aprendizagem.

Em idade escolar, crianças com quadros "típicos" de EAI podem ser tratadas como portadoras de "déficit de atenção" podendo ser encaminhados ao atendimento neurológico com queixa de "baixo rendimento na escola". Este equívoco leva a um diagnóstico e intervenção tardia, podendo contribuir para maiores danos e sequelas à essas crianças. Outro aspecto a ser considerado é o diagnóstico diferencial entre crises de ausência e crises parciais complexas, evitando-se o uso antiepilépticos não efetivos no tratamento de crianças com EAI.

Para que haja bom desempenho escolar, é fundamental que os pais, professores e profissionais envolvidos no diagnóstico aliem-se, propiciando à criança um ambiente de aceitação e tratamento adequado, onde possa desenvolver-se adequadamente.

\section{REFERÊNCIAS}

1.Acharya M, Hattiangdy B, Shetty A. Progress in neuroprotective strategies for preventing epilepsy. Prog Neurobiol 2008;84:363-404.

http://dx.doi.org/10.1016/j.pneurobio.2007.10.010

2.Schilndwein-zanini R. Liguagem e Cognição da criança com epilepsia no contexto educacional. Atos Pes Edu 2011;6:245-51.

3.Noronha ALA, Borges A, Marques LH, Zanetta DMT, Fernandes PT, Boer $\mathrm{H}$, et al. Prevalence and patternoof epilepsy treatment in different social-economic classes in Brazil. Epilepsia 2007;8:880-5.

http://dx.doi.org/10.1111/j.1528-1167.2006.00974.x

4.Brodie M, Schachter S, Kwan P. Fast Facts: Epilepsy. 3rd ed. Oxford: Health Press; 2008, 144p.

5.Glauser TA, Cnaan A, Shinnar S, Hirtz DG, Dlugos D, Masur D, et al. Ethosuximide, valproic acid, and lamotrigine in childhood absence Epilepsy. N Engl J Med 2010;362:790-9.

http://dx.doi.org/10.1056/NEJMoa0902014

6.Nadler B, Shevell MI. Childhood absence epilepsy requiring more than one medication for seizure control. Can J Neurol Sci 2008;35:297-300.

7.Farooque P, Goraya J, Valencia I, Carvalho KS, Hardison HH, Legido A, et al.Early-onset childhood absence epilepsy: is it a distinct entity? Epileptic Disord 2011;13:411-6.

8.Ponnusamy A, Rao G, Baxter P, Field P. Ictal hiccup during absence seizure in a child. Epileptic Disord 2008;10:53-5.

9.Tanaka M, Olsen RW, Medina MT, Schwartz E, Alonso ME, Duron RM, et al. Hyperglycosylation and reduced GABA currentsof mutated GABRB3 polypeptidein remitting childhood absence epilepsy. Am J Hum Gen 2008;82:1249-61.

http://dx.doi.org/10.1016/j.ajhg.2008.04.020 
10.Gibbs FA, Davis H, Lennox WG. The EEG inepilepsy and in conditions of impaired consciousness. Arc Neurol Psychiatr 1935;34:1133-48.

http://dx.doi.org/10.1001/archneurpsyc.1935.02250240002001

11.Grippo J, Grippo TM. Canalopatíasen Neurología: síndromes epilépticos idiopáticos. Arc Arg Ped 2007;105:447-52.

12.García-Peñas JJ. Interictal epileptiform discharges and cognitive impairment in children. Rev Neurol 2011;52(Suppl 1):S43-52.

13.Berg AT. Epilepsy, cognition, and behavior: the clinical picture. Epilepsia 2011;52(Suppl 1):7-12.

http://dx.doi.org/10.1111/j.1528-1167.2010.02905.x

14.Pardoe H, Pell GS, Abbott DF, Berg AT, Jackson GD. Multi-sitevoxel-based morphometry: methods and a feasibility demonstrationwith childhood absence epilepsy. Neuroimage 2008;42:611-6.

http://dx.doi.org/10.1016/j.neuroimage.2008.05.007

15.Paula GR, Beber BC, Baggio SB, Petry T. Neuropsicologia da aprendizagem. Rev Psicopedag 2006;23:224-3.

16.Alberto I. Atenção, por favor (!) à avaliação da atenção. Psychologica 2003;34:231-43.

17.Lopes AFD, Simôes MMR, Robalo CN, Fineza I, Gonçalves OB. Evaluación neuropsicológica enniñoscon epilepsia: atención y funciones ejecutivasen epilepsia del lóbulo temporal. Rev Neurol 2010;265:72.

18.ÁIdiazábal-Alecha A, Kosno M. Transtorno por déficit de atención/hiperactividad y epilepsia em la infância. Rev Neurol 2012;54(Supl 1):S89-93.

19.D’Agati E, Cerminara C, Casarelli L, Pitzianti M, Curatolo P. Attention and executive functions profile in childhood absence epilepsy. Brain Develop 2012;34:812-7.

http://dx.doi.org/10.1016/j.braindev.2012.03.001

20.Jeong MH, Yum MS, Ko TS, You SJ, Lee EH, Yoo HK. Neuropsychological status of children with newly diagnosed idiopathic childhood epilepsy. Brain Develop 2011;33:666-71.

http://dx.doi.org/10.1016/j.braindev.2010.11.003
21.Dunn DW, Kronenberger WG. Psychiatric disorders in epilepsy: attention-deficit hyperactivity disorder, attention problems and epilepsy. In: Ettinger AB; Kanner AM. Psychiatric issues in epilepsy: a practicalguide to diagnosis and treatment. Philadelphia: Lippincott Williams \& Wilkins, 2007, p.272-85. 22.Serardi A, Tsalkidis A, Tripsianis G, Vadikolias K, Chatzimichael A, Piperidou C, et al. Epilepsy impact on aspects of school life of children from different cultural populations in Thrace, Greece. Epilepsy Beh 2009;15:344-50.

http://dx.doi.org/10.1016/j.yebeh.2009.04.025

23.MacAllister WS, Schaffer SG. Neuropsychological deficits inchildhood epilepsy syndromes. Neuropsychol Rev 2007;17:427-44.

http://dx.doi.org/10.1007/s11065-007-9048-4

24.Aldenkamp A, Rejis R, Mil V, Hall M. Attention disorders in children with epilepsy. Epilepsia 2005;46(Suppl 6):S6.

25.Aguiar BVK, Guerreiro MM, McBrian D, Montenegro MA. Seizure impact on the school attendance in children with epilepsy. Seizure 2007;16:698-702. http://dx.doi.org/10.1016/j.seizure.2007.05.013

26. Bhise VV, Burack GD, Mandelbaum DE.Baseline cognition,behavior, and motor skills in children with new-onset, idiopathicepilepsy. Dev Med Child Neurol 2010;52:22.

http://dx.doi.org/10.1111/j.1469-8749.2009.03404.x

27.Eddy CM, Rickards EH, Cavanna AE. The cognitive impact of antiepileptic drugs. Ther Adv Neurol Disord. 2011;4:385-407.

http://dx.doi.org/10.1177/1756285611417920

28.Caplan R, Siddarth P, Stahl L, Lanphier E, Vona P, Gurbani S, et al. Childhood absence epilepsy: behavioral, cognitive, and linguistic comorbidities. Epilepsia 2008;49:1838-46.

http://dx.doi.org/10.1111/j.1528-1167.2008.01680.x

29.Jones JE, Siddarth P, Gurbani S, Shields WD, Caplan R. Cognition, academic achievement, language, and psychopathology in pediatric chronic epilepsy: Short-term outcomes. Epilepsy Behav 2010;18:211-7.

http://dx.doi.org/10.1016/j.yebeh.2010.03.015 